and personal air sampling pump equipped with XAD-2 filters. In parallel, we performed detailed observations of work characteristics on the whole day and measured residues on various surfaces.

Results We observed 30 treatment days corresponding to 52 mixing/loading, 52 spraying and 12 equipment cleaning tasks. The median daily dermal contamination was $5.5 \mathrm{mg}$ for captan and $3.3 \mathrm{mg}$ for dithianon. The average contribution of each phase to the daily dermal contamination was $41.5 \%$ for mixing/loading, $29.6 \%$ for spraying and $33.0 \%$ for cleaning. During mixing/loading, the major determinants of the contamination were related to the use of gloves, the product handling and the characteristics of the spraying equipment. During spraying, dermal contamination was strongly correlated to the spraying equipment (e.g. type and age of the sprayer) and the characteristics of the orchard (height of trees, distance between rows). The daily contamination was also correlated to these parameters.

Conclusion Key determinants of exposure will be used to build prevention messages to improve the safety of operators during treatment operations, especially during mixing and cleaning, the most contaminant tasks. They will also be relevant to build questionnaires for epidemiological studies.

\section{P-155 OCCUPATIONAL EXPOSURE TO ORGANOCHLORINE INSECTICIDES AND PROSTATE CANCER RISK IN THE AGRICULTURE AND CANCER (AGRICAN) COHORT}

${ }^{1}$ Marine Morin Renier, Clémentine Lemarchand, Séverine Tual, Mathilde Bureau, Stephanie Perrier, Anne-Valérie Guizard, Elisabeth Marcotullio, Isabelle Baldi, Pierre Lebailly. ${ }^{1}$ Centre François Baclesse, France

\subsection{6/OEM-2021-EPI.213}

Introduction Prostate cancer incidence ranks 2nd among men worldwide. Farming and pesticide use have been repeatedly and consistently associated with prostate cancer risk, with suggestion of the role of organochlorine pesticides, but results on individual pesticides remain controversial.

Objectives We assessed associations between prostate cancer and agricultural exposure to organochlorine insecticides (as a group and considering individual pesticides: aldrin, chlordane, $\mathrm{HCH}$, dieldrin, lindane, heptachlore, DDT, DDD, endosulfan, metoxychlore, SPC, toxaphen, chlorfenethol, chlorobenzilate, dicofol, bromopropylate).

Methods Data on pesticide use on 10 crops, including years of beginning and ending, were collected for 81,960 men from the enrolment questionnaire of AGRICAN. Incident prostate cancer cases were identified through linkage with cancer registries. Exposure to organochlorine insecticides was assessed using the crop-exposure matrix PESTIMAT. Hazard Ratios (HR and 95\% CI) were estimated using Cox models with attained age as time scale.

Results Until 2015, 3,535 prostate cancers were diagnosed. We found a slight increase in the risk of prostate cancer in men diagnosed until 2009 exposed to organochlorines $(\mathrm{HR}=1.11$ [0.96-1.30]), but the trend disappeared with 6 additional years of follow-up $(\mathrm{HR}=0.95[0.87-1.05])$. This result was also globally found when considering individual pesticides. But for substances still in use after 1980 (lindane, dicofol, endosulfan, toxaphen), some increases were still observed with longer follow-up: for lindane used on wheat/barley for $\geq 40$ years $(\mathrm{HR}=1.27[1.01-1.58])$, on rape for $\geq 30$ years $(\mathrm{HR}=1.52$ [0.86-2.70]), on sunflower for $\geq 10$ years $(\mathrm{HR}=1.14[0.87-$ 1.51]) and on vineyard for $\geq 30$ years ( $\mathrm{HR}=1.09[0.90-1.32])$, for dicofol used $\geq 40$ years on vineyards $(\mathrm{HR}=1.17[0.89$ $1.53])$, and for endosulfan used on fruit-growing $(\mathrm{HR}=1.16$ [0.95-1.41]).

Conclusions The increased risk of prostate cancer associated with organochlorines initially observed tended to disappear with follow-up duration, except for some substances still in use in the 1980s and 1990s. Explanations could be i) the distance between last exposure for some organochlorines and cancer diagnosis, ii) changes in the exposure intensity due to evolution of practices.

\section{P-164 WORK-RELATED MUSCULOSKELETAL DISORDERS OF THE UPPER LIMB IN PRACTIONERS IN THE DENTAL TEACHING CLINIC OF TUNISIA.}

1Ons Grissa, Marouen Hayouni, Imen El Kharras, Ines Rassas, Charfeddine Amri, Neila Chaari, Adnene Henchi, Mohamed Akrout, Mohamed ben Khlifa, Irtyah Merchaoui. ${ }^{1}$ Faculty of Medicine of Monastir, University of Monastir, Tunisia

\subsection{6/OEM-2021-EPI.214}

Introduction Musculoskeletal disorders represent an important occupational health issue in Dentistry. Awkward postures as well as repetitive motion and the lack of ergonomic tools and workstation expose to a variety of musculoskeletal disorders affecting dentists' quality of life. Therefore, ergonomic recommendations should be applied since the beginning of the university teaching and training.

Objectives The aims of our study were to assess the prevalence of upper limb MSDs among dental practitioners in the dental teaching clinic of Monastir and to identify the social, occupational, ergonomic, and psychosocial risk factors of their occurrence.

Methods It is a cross-sectional study, that interested 260 dental practitioners working in the hospital academic center of dentistry. It was based on a self-administered questionnaire exploring the socio-demographic, occupational and ergonomic data. It was completed by the standardized Nordic Questionnaire and Karasek one.

Results Ninety six percent $(96.4 \%)$ of the participants reported upper limb MSDs including 95.2\% with multifocal locations. The most common painful site was the elbow. The presence of upper limb MSDs was significantly correlated with a high number of patients under care $(p=10-3)$, better social support $(p=0.04)$, single status $(p=0.03)$ and the position as a dental trainee $(p=0.006)$. There is no significant difference in terms of upper limb MSD between subjects in a Job Strain situation and those who were not. Furthermore, no ergonomic determinants were found with the advent of upper limb MSDs.

Conclusion Upper limb MSDs risk among dental practitioners at the dental academic center of dentistry of Monastir is real and frequent. Prevention of this occupational issue should include ergonomic workshops in dental university course and awareness risk sessions. Prevention is obviously based on tools, movement, and space Ergonomics. 